\title{
MGr1-Ag promotes invasion and bone metastasis of small-cell lung cancer in vitro and in vivo
}

\author{
FENG ZHANG $^{1^{*}}$, YANXIA WANG ${ }^{2 *}$, MIN XU $^{2}$, HAIYING DONG ${ }^{2}$, NA LIU $^{1}$, JING ZHOU $^{1}$, HAILIN PANG ${ }^{1}$, \\ NINGQIANG MA ${ }^{1}$, NING ZHANG ${ }^{1}$, YANLIN PEI ${ }^{1}$, HELONG ZHANG ${ }^{1}$ and LILI LIU ${ }^{1}$ \\ ${ }^{1}$ Department of Oncology, Tangdu Hospital, The Fourth Military Medical University, Xi'an 710038; \\ ${ }^{2}$ Department of Pathology and Pathophysiology, The Fourth Military Medical University, Xi'an 710032, P.R. China
}

Received February 19, 2013; Accepted March 14, 2013

DOI: $10.3892 /$ or.2013.2396

\begin{abstract}
Bone metastasis of small-cell lung cancer (SCLC) usually occurs early in the progression of the disease. However, the molecular mechanism underlying bone metastasis is largely unknown. MGr1-Ag, a multifunction protein, has been suggested to play important roles in cell growth, differentiation and migration. In our present study, MGrl-Ag was found to be highly expressed in bone-metastatic SCLC cells (SBC-5 cell line) as compared with the expression in cells without bonemetastatic ability (SBC-3 cell line). Therefore, we hypothesized that MGr1-Ag is involved in bone metastasis of SCLC. Using a sense vector to upregulate MGr1-Ag expression in SBC-3 cells, we found that forced overexpression of MGr1-Ag enhanced cell invasion and migration in vitro and promoted bone metastases in vivo. Furthermore, specific siRNA-induced knockdown of MGr1-Ag expression in SBC-5 cells suppressed the potential of cell invasion and migration in vitro and dramatically decreased the number and sites of bone metastasis in vivo. We also found that MGr1-Ag induced SCLC cells to undergo epithelial-mesenchymal transition (EMT), as demonstrated by cell morphological changes, decreased expression of epithelial markers and increased expression of mesenchymal markers. Taken together, we conclude that MGr1-Ag promotes SCLC cell invasion and bone metastasis in vitro and in vivo, and that this is partially mediated via the EMT pathway.
\end{abstract}

\section{Introduction}

Globally, lung cancer is the major cause of malignancy-related mortality, and its incidence is on the rise in many countries (1).

Correspondence to: Professor Lili Liu or Professor Helong Zhang, Department of Oncology, Tangdu Hospital, The Fourth Military Medical University, No. 1 Xinsi Road, Xi'an 710038, P.R. China

E-mail: 1ily123joe@yahoo.com.cn

E-mail: cnxazhl@163.com

*Contributed equally

Key words: small-cell lung cancer, MGr1-Ag, bone metastasis
Histologically, lung cancer is classified as small-cell lung cancer (SCLC) and non-small cell lung cancer (NSCLC). Due to the high ability of rapid growth and early distant metastasis, the prognosis of SCLC is considered be the poorest of all lung cancer types. Its 5-year survival rate is less than $2 \%(2,3)$. Approximately two-thirds of SCLC patients present obvious metastatic disease (4). In particular, bone is one of the most common sites of metastasis. Osteolytic metastases are incurable and are often associated with skeletal-related events including pain, hypercalcemia, fracture and nerve compression syndromes, all of which decrease the quality of life of patients (5). However, the mechanism underlying the bone metastatic capacity of SCLC remains obscure. Bone metastasis occurs as a sequence of complex processes involving multiple interactions of cancer cells with host cellular and extracellular microenvironments. Various molecules, such as adhesion molecules, cytokines (6), chemokines $(7,8)$, hormones (9) and their receptors have been reported to play important roles in these processes.

SBC-3 and SBC-5 cell lines demonstrate a similar genetic background to human SCLC but a different potential for bone metastasis. SBC-5 has a higher capability of bone metastasis than SBC-3 (10-12). Consequently, this pair of cell lines is widely used as cell models in the research of the molecular pathogenesis of bone metastasis in human SCLC.

In our laboratory, we previously explored the preliminary mechanism of bone metastasis using SCLC cell lines (SBC-3 and SBC-5). We found that calcineurin $\mathrm{A} \alpha$ and zinc finger E-box binding homeobox 1 (ZEB1) were closely related to the osteotropic metastasis of SCLC. In addition, the epithelialmesenchymal transition (EMT) pathway was observed to participate in the process of bone metastasis in SCLC (13-15). However, the exact mechanism is still unknown.

MGr1-Ag, also termed as the 37-kDa laminin receptor precursor (37LRP), is the precursor of the metastasis-associated $67-\mathrm{kDa}$ laminin receptor (67LR). It exhibits high lamininbinding activity and appears to be a ribosomal protein which is essential for protein synthesis $(16,17)$. Increased research interest has been stimulated by the observation that MGrl-Ag is a multifunctional protein that is required for cell differentiation, migration and growth. We and other researchers have consistently observed it in invasive and metastatic cancers, and it is associated with poor prognosis (18-20). Therefore, we 
proposed that MGr1-Ag may play a role in the process of bone metastasis in SCLC.

EMT has been proposed as a key step during carcinoma progression and metastatic development. Its hallmark is the loss of polarized organization and downregulation of epithelial molecular markers (e.g. E-cadherin, $\alpha$-catenin, and $\beta$-catenin), and at the same time, upregulation of mesenchymal proteins (e.g. fibronectin, sm-actin and vimentin) $(21,22)$. According to previous research, the worst prognosis of SCLC is associated with the presence of cells with a mesenchymal character (23). This indicates that EMT plays an important role in SCLC cell metastasis.

In the present study, we first observe that MGr1-Ag was highly expressed in bone-metastatic SCLC cell line SBC-5. Subsequently we investigated the effects of the upregulation or knockdown of MGr1-Ag expression on cell invasion and migration in vitro as well as the bone metastatic ability of SCLC cell lines in vivo. Additionally, changes in cell morphology and the expression of EMT markers (E-cadherin and fibronectin) were observed. The results demonstrated that MGrl-Ag contributes to an EMT-like transformation, invasion and metastasis in bone-metastatic SCLC. Therefore, we present initial evidence that MGrl-Ag promotes the occurrence of bone metastasis in SCLC via the EMT pathway.

\section{Materials and methods}

Cell culture. The human SCLC cell lines SBC-3 and SBC-5 were gifts from Professor Saburo Sone and Seiji Yano (University of Tokushima School of Medicine, Japan) (11). The human lung fibroblast (HLF) cell line, used as a control, was from the Shanghai Cell Bank (Shanghai, China). The cells were cultured in RPMI-1640 medium (Gibco, Carlsbad, CA, USA) containing $100 \mathrm{U} / \mathrm{ml}$ penicillin, $100 \mu \mathrm{g} / \mathrm{ml}$ streptomycin and $10 \%$ fetal calf serum (FCS). Cultures were incubated in a humidified atmosphere with $5 \% \mathrm{CO}_{2}$ at $37^{\circ} \mathrm{C}$.

Plasmid constructs and transfection. To upregulate and downregulate the expression of MGr1-Ag in the SCLC SBC-3 and SBC-5 cell lines. MGr1-Ag-targeting oligonucleotides for generating cDNA were designed from full-length MGrl-Ag by Shanghai GeneChem Co., Ltd. Their sequences were forward, 5'-GAGGATCCCCGGGTACCGGTCGCCACCATGCCGGA GCCCTTGAT-3' and reverse, 5'-TCACCATGGTGGCGACCG GAGACCAGTCAGTGGTTGCTC-3'. Three pairs of MGr1Ag-specific siRNAs were also designed (Shanghai GeneChem Co., Ltd.): MGr1-Ag siRNA1 forward, 5'-cgggaGATCCTG AAGAGATTGAAATTCAAGAGATTTCAATCTCTTCAGG ATCtcTTTTTg-3' and reverse, 5'-aattcaaaaagaGATCCTGAA GAGATTGAAATCTCTTGAATTTCAATCTCTTCAGGAT Ctc-3'; siRNA2 forward, 5'-CcggcaCTCCTGGAACCTTCACT AATTCAAGAGATTAGTGAAGGTTCCAGGAGtgTTTTg-3' and reverse, 5'-aattcaaaaacaCCTCTTGAATTAGTGAAGGTT CCAGGAGtg-3'; siRNA3 forward, 5'-CcggtaCCTACCATTGC GCTGTGTATTCAAGAGATACACAGCGCAATGGAGGtaT TTTTg-3' and reverse, 5'-aattcaaaaataCCTACCATTGCGCTG TGTATCTCTTGATACACAGCGCAATGGTAGGta-3'. After testing overexpression and knockdown efficiencies, stem-loop oligonucleotides were synthesized and cloned into the lentivirus-based vector pSicoR (Addgene). A non-targeting (scram- bled) stem-loop DNA pSicoR vector was generated as a negative control. Lentiviral particles were prepared as described previously (24). The overexpression and knockdown efficiencies were then confirmed by western blot analysis and real-time PCR. SBC-3 cells transfected with MGr1-Ag cDNA or empty vector were designated SBC-3/MGr and SBC-3/pc. SBC-5 cells transfected with siRNA-lentivirus-MGr1-Ag or scrambled RNA duplex were designated SBC-5/MGr-si and SBC-5/scr.

Real-time reverse transcription $(R T)$-polymerase chain reaction $(P C R)$. Total cellular RNA was extracted using TRIzol reagent from the cells following the different treatments, and was then reverse transcribed using the SuperScript ${ }^{\circledR}$ III RT-PCR system (both from Invitrogen, USA), according to the manufacturer's instructions. Synthesized cDNA was used as a template for the TaqMan real-time PCR technique to quantify mRNA expression by using a qPCR core kit (Eurogentec) and an ABI Prism 7700 sequence detection system (PE Applied Biosystems). TaqMan PCR primers for each gene were designed as in a previous study (20). The primer sequences included: $\beta$-actin forward, 5'-GGCGGCACCACCATGTACCCT-3' and reverse, 5'-AGGGGCCGGACTCGTCATACT-3'; MGr1-Ag forward, 5'-GCAGCAGGAACCCACTTAGG-3' and reverse, 5'-GGCAGCAGCAAACTTCAGC-3'.

Western blot analysis. After pretreatment according to experimental demand, the cells were washed three times with ice cold phosphate-buffered saline (PBS). Then, the cytosolic extracts were extracted with lysis buffer $(62.5 \mathrm{mM}$ Tris- $\mathrm{HCl}, \mathrm{pH} 6.8$, $2 \%$ SDS, 5\% 2-mercaptoethanol, $10 \%$ glycerol and protease inhibitor cocktail). According to the standard protocol for western blot analysis, equal amounts of protein $(50 \mu \mathrm{g})$ were separated by $12 \%$ SDS-polyacrylamide gel electrophoresis (SDS-PAGE) and electrotransferred onto polyvinylidene difluoride (PVDF) membranes. The proteins were hybridized with primary antibodies against MGr1-Ag (1:200; developed by Bioss Biotech, Beijing, China), E-cadherin (1:500), fibronectin (1:500; both from BD Biosciences, Franklin Lakes, NJ, USA) or $\beta$-actin (1:4,000; Sigma, USA). After staining with an HRP-linked secondary antibody (GE Healthcare Bio-Science, USA), the protein bands were detected by the ECL chemiluminescence system (Amersham Phamacia Biotech, USA).

In vitro invasion and migration assays. In vitro cell migration assays were performed as described previously (13) using Transwell chambers ( $8-\mu \mathrm{m}$ pore size; Costar). Cells were allowed to grow to $\sim 75-80 \%$ confluence and were serum-starved for $24 \mathrm{~h}$. After detachment with trypsin, cells were washed with PBS, resuspended in serum-free medium, and $250 \mu \mathrm{l}$ of the cell suspension $\left(2 \times 10^{5}\right.$ cells $\left./ \mathrm{ml}\right)$ was added to the upper chamber. Complete medium was added to the bottom wells of the chamber. The cells that had not migrated were removed from the upper face of the filters using cotton swabs, and the cells that had migrated to the lower face of the filters were fixed with $5 \%$ glutaraldehyde solution and stained with $0.5 \%$ solution of toluidine blue in $2 \%$ sodium carbonate. Images of three random x10 fields were captured from each membrane, and the number of migratory cells was counted. The mean of triplicate assays for each experimental condition was used. Similar inserts coated with Matrigel (BD 
Biosciences) were used to determine the invasive potential in the invasion assay.

Wound healing assay. The wound healing assay was performed as described previously (25). In brief, cells were seeded in 6 -well plates and allowed to grow to $\sim 90 \%$ confluence before wounding with a $200 \mu \mathrm{l}$ plastic tip across the monolayer cells. Debris was removed by washing three times with PBS, and then cells were cultured with fresh medium containing $5 \%$ fetal bovine serum. After $48 \mathrm{~h}$, images were captured by phase-contrast microscope. Each experiment was performed in triplicate and repeated three times.

Immunofluorescence. Cells transfected with the indicated expression vectors were plated on sterile microscope coverslips. After $24 \mathrm{~h}$, cells were washed and fixed in acetone/ methanol (1:1) solution for $3 \mathrm{~min}$ on ice. Then the coverslips were rinsed and incubated with primary antibodies specific for E-cadherin or fibronectin (1:500; BD Biosciences) for $1 \mathrm{~h}$ at room temperature. After washing, the coverslips were incubated with Alexa 488-conjugated rabbit anti-mouse IgG (Pierce Biotechnology, Inc., Rockford, IL, USA) for $1 \mathrm{~h}$. Finally, cells were examined and photographed using a confocal inverted microscope (Axiovert 200M; Zeiss, Oberkochen, Germany). To monitor BrdU incorporation, cells were pulse labeled with BrdU for $40 \mathrm{~min}$ and stained with DAPI according to the protocols supplied with the Detection Kit I (Roche Applied Science, Basel, Switzerland).

Tail-vein bone metastatic assay. Female NOD/scid mice, 3-4 weeks old, were purchased from HFK Bioscience Co., Ltd. (Beijing, China) and maintained in our animal facilities under specific pathogen-free conditions. The mice were divided into groups and injected through the tail vein with $1 \times 10^{6}$ cells in $0.1 \mathrm{ml}$ PBS and monitored for overall health and total body weight. Five weeks after injection, the mice were sacrificed. The number of sites of bone metastases on the body surface was counted. Sites of bone metastases were serially sectioned for X-ray analysis and observed under a light microscope. Each experimental group contained 5 mice. All animals were handled using best humane practices and cared for in accordance with National Institutes of Health Animal Care and Use Committee guidelines.

Statistical analysis. Statistical analysis was performed using SPSS software (version 11.0; Chicago, IL, USA). Assays for characterizing cell phenotypes were analyzed using Student's t-test. The Wilcoxon rank sum test was used to determine the significance of differences in bone metastasis numbers between groups. $\mathrm{P}<0.05$ was considered to indicate a statistically significant result.

\section{Results}

MGrl-Ag is overexpressed in the bone-metastatic SCLC cell line. Previous studies have shown that the SCLC cell lines, SBC-3 and SBC-5, have similar genetic background but different potential for bone metastasis. SBC-5 has significantly higher capability of bone metastasis than SBC-3 (10,11). To detect the differential expression of MGr1-Ag in these cell
A

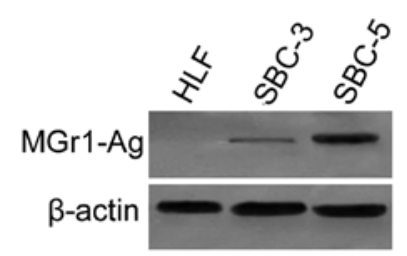

B

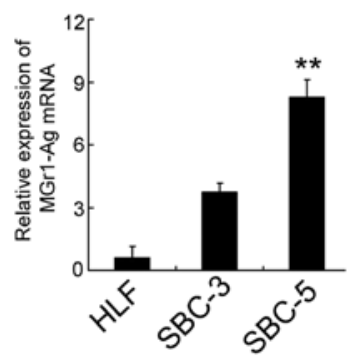

Figure 1. Expression analyses of MGr1-Ag in bone-metastatic and non-bonemetastatic SCLC cells. (A) Expression of MGrl-Ag at the protein level was detected by western blot analysis. In this and other experiments, $\beta$-actin was used as a loading control. (B) Expression of MGr1-Ag at the mRNA level was detected by real-time PCR ( ${ }^{* *} \mathrm{P}<0.01$ vs. SBC-3).

lines, western blot analysis and real-time PCR were performed. Human lung fibroblasts (HLFs) (normal cells without bonemetastatic ability) were used as the negative control in each experiment. Data from the western blot analysis showed that the protein expression level of MGrl-Ag in SBC-5 cells was distinguishably higher than that in the SBC-3 cells (Fig. 1A). Similarly, the differential expression of MGrl-Ag mRNA was confirmed by data from real-time PCR ( $\mathrm{P}<0.01$ vs. SBC-3) (Fig. 1B). These results indicated that MGr1-Ag overexpression was closely associated with the bone-metastatic ability of SCLC cells.

Upregulated expression of MGrl-Ag in SBC-3 cells enhances cell invasion and migration in vitro, and increases the number of bone metastases in vivo. To further explore the effect of MGr1-Ag on bone metastasis in SCLC, MGr1-Ag stably transfected cell line SBC-3/MGr was constructed according to Materials and methods. The SBC-3/pc cell line was employed as the control-transfected cells. Western blot analysis and real-time PCR were performed to test the efficiencies of MGr1-Ag upregulation. The data revealed that MGr1-Ag expression was markedly increased in the $\mathrm{SBC}-3 / \mathrm{MGr}$ cells when compared with that in $\mathrm{SBC}-3 / \mathrm{pc}$ cells at the protein and mRNA levels, respectively $(\mathrm{P}<0.01$ vs. SBC-3/pc) (Fig. 2A and B). Subsequently, we detected the invasive and migratory ability of each cell group in vitro. The results from the Transwell assays showed that SBC-3/MGr cells demonstrated increased migratory and invasive abilities compared with the control cells $(\mathrm{P}<0.01$ vs. SBC-3/pc) (Fig. 3A and C). Meanwhile, data from the wound healing assay revealed that, compared with SBC-3/pc cells, SBC-3/MGr cells exhibited a much greater migratory capacity to repair the wound (Fig. 3E-a and -b).

Furthermore, tail-vein bone metastatic assays were adopted to examine the bone-metastatic ability of SBC-3, $\mathrm{SBC}-3 / \mathrm{pc}$ and SBC-3/MGr cells in vivo. Compared with control cells (SBC-3/pc), the injection of SBC-3/MGr cells led to a significant increase in bone metastatic lesions $(\mathrm{P}<0.01$ vs. SBC-3/pc) (Table I and Fig. 3F-a and -b). Taken together, these results suggest that $\mathrm{MGrl}-\mathrm{Ag}$ overexpression promotes the invasion, migration and bone-metastatic abilities of SCLC cells both in vitro and in vivo. 


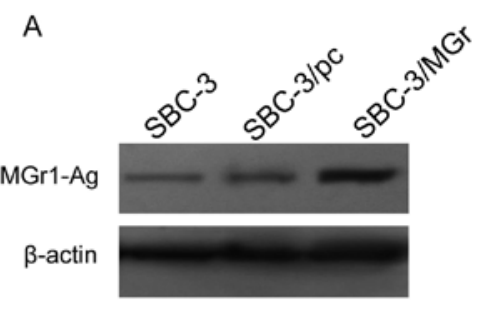

B

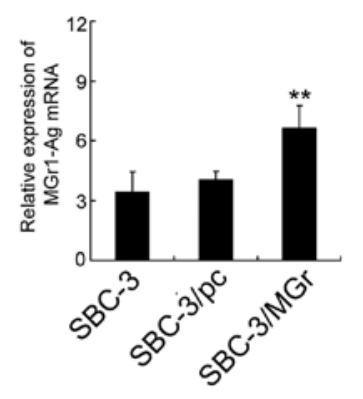

C

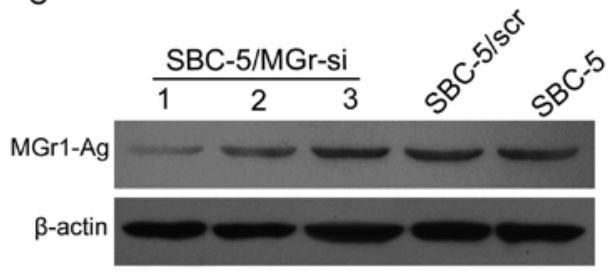

D

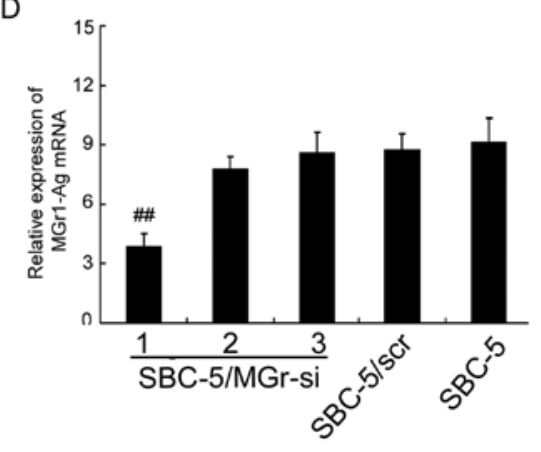

Figure 2. Expression analyses of MGr1-Ag in SCLC cells transfected with the MGrl-Ag sense vector or the siRNA vector. The SBC-3 cell line was transfected with MGr1-Ag sense vector and the SBC-5 cell line was transfected with a specific siRNA vector targeting MGr1-Ag. (A and B) The protein and mRNA expression levels of MGr1-Ag in SBC-3, SBC-3/pc and SBC-3/ MGr cells were detected by western blot analysis or real-time PCR, respectively. SBC-3/pc cells were used as a control in this and other experiments $\left({ }^{* *} \mathrm{P}<0.01 \mathrm{vs}\right.$. SBC-3/pc). (C and D) The protein and mRNA expression levels of MGr1-Ag in SBC-5, SBC-5/scr and SBC-5/MGr-si cells were detected by western blot analysis or real-time PCR, respectively. SBC-5/scr cells were used as control in this and other experiments $(\# / \mathrm{P}<0.01$ vs. SBC-5/scr).

Suppressed expression of MGrl-Ag in SBC-5 cells inhibits cell invasion and migration in vitro, and decreases the number of bone metastases in vivo. To further confirm the effect of MGr1-Ag in bone metastasis of SCLC, RNA interference was employed to knockdown the expression of MGr1-Ag in the SBC-5 cell line. The knockdown efficiency was confirmed by western blot analysis and real-time PCR. MGr1-siRNA1 effectively downregulated MGr1-Ag in SBC-5 cells at the protein and mRNA level, while the effect of
Table I. Bone metastatic lesions in the NOD/scid mice model after inoculation of the indicated cells through the tail vein.

\begin{tabular}{lcc}
\hline Group & Incidence & Median (range) \\
\hline SBC-3 & $0 / 5$ & $0(0-0)$ \\
SBC-3/pc & $0 / 5$ & $0(0-0)$ \\
SBC-3/MGr & $4 / 5$ & $3(0-5)^{\mathrm{a}}$ \\
SBC-5 & $5 / 5$ & $9(8-12)$ \\
SBC-5/scr & $5 / 5$ & $11(8-13)$ \\
SBC-5/MGr-si & $3 / 5$ & $4(0-7)^{\mathrm{b}}$ \\
\hline
\end{tabular}

The colonies of metastasis to bone were counted. ${ }^{\mathrm{a}} \mathrm{P}<0.01 \mathrm{vs}$. SBC-3/pc group; ${ }^{\text {b }}<0.01$ vs. $\mathrm{SBC}-5 /$ scr group (Wilcoxon rank sum test).

MGr1-siRNA2 and MGr1-siRNA3 was minima ( $\mathrm{P}<0.01$ vs. SBC-5/scr) (Fig. 2C and D). Thus, SBC-5 cells transfected with siRNA1 (termed SBC-5/MGr-si) were used in the subsequent investigations. SBC-5/scr cells were used as control. Next, cell invasion and migration in the in vitro assays showed that downregulation of MGr1-Ag in SBC-5 cells significantly inhibited their migratory and invasive abilities compared with control cells ( $\mathrm{P}<0.01$ vs. SBC-5/scr) (Fig. 3B and D). The wound healing assay was also performed in SBC-5 cells. As shown in Fig. 3E-c and -d, knockdown of MGrl-Ag notably reduced the migratory capacity of SBC-5 cells to repair the wound. Meanwhile, to investigate the effect of MGr1-Ag knockdown on the ability of bone metastasis, SBC-5/MGr-si cells were injected into NOD/scid mice and assayed for the development of bone metastatic lesions. Compared with the control group, the injection of SBC-5/MGr-si cells led to a significant decrease in the number of bone metastatic lesions $(\mathrm{P}<0.01$ vs. SBC-5/scr) (Table I and Fig. 3F-c and $-\mathrm{d}$ ). These results suggest that, both in vitro and in vivo, inhibition with siRNA targeting MGrl-Ag had the potential to suppress the invasion, migration and bone-metastatic ability of SCLC cells.

EMT is involved in MGrl-Ag induced bone metastasis in $S C L C$. The cell morphology of most SBC-3 cells which have no bone-metastatic ability was round, while that of SBC-5 cells which have high bone-metastatic ability was fusiform. Apart from this, after transfection with the MGr1-Ag overexpression vector, we noted that the morphology of SBC-3/MGr cells changed from round to fusiform (Fig. 4A). Yet, in the SBC-5 cells, the cell morphology was altered from fusiform to round after the inhibition of MGr1-Ag expression with siRNA (Fig. 5A). These differences and changes represent one of the hallmarks of EMT and indicated that EMT was involved in the occurrence of bone metastases in SCLC, and that MGrl-Ag induced EMT in the SCLC cell lines. To gain insight into the mechanism of MGrl-Ag-induced EMT, we next examined the expression of well-characterized EMT markers. Immunofluorescence staining showed that the epithelial marker E-cadherin had nearly disappeared and the mesenchymal marker fibronectin was dramatically increased in the SBC-3/MGr cells compared to the staining observed in the control cells (Fig. 4C). Western blot analysis revealed nearly a 

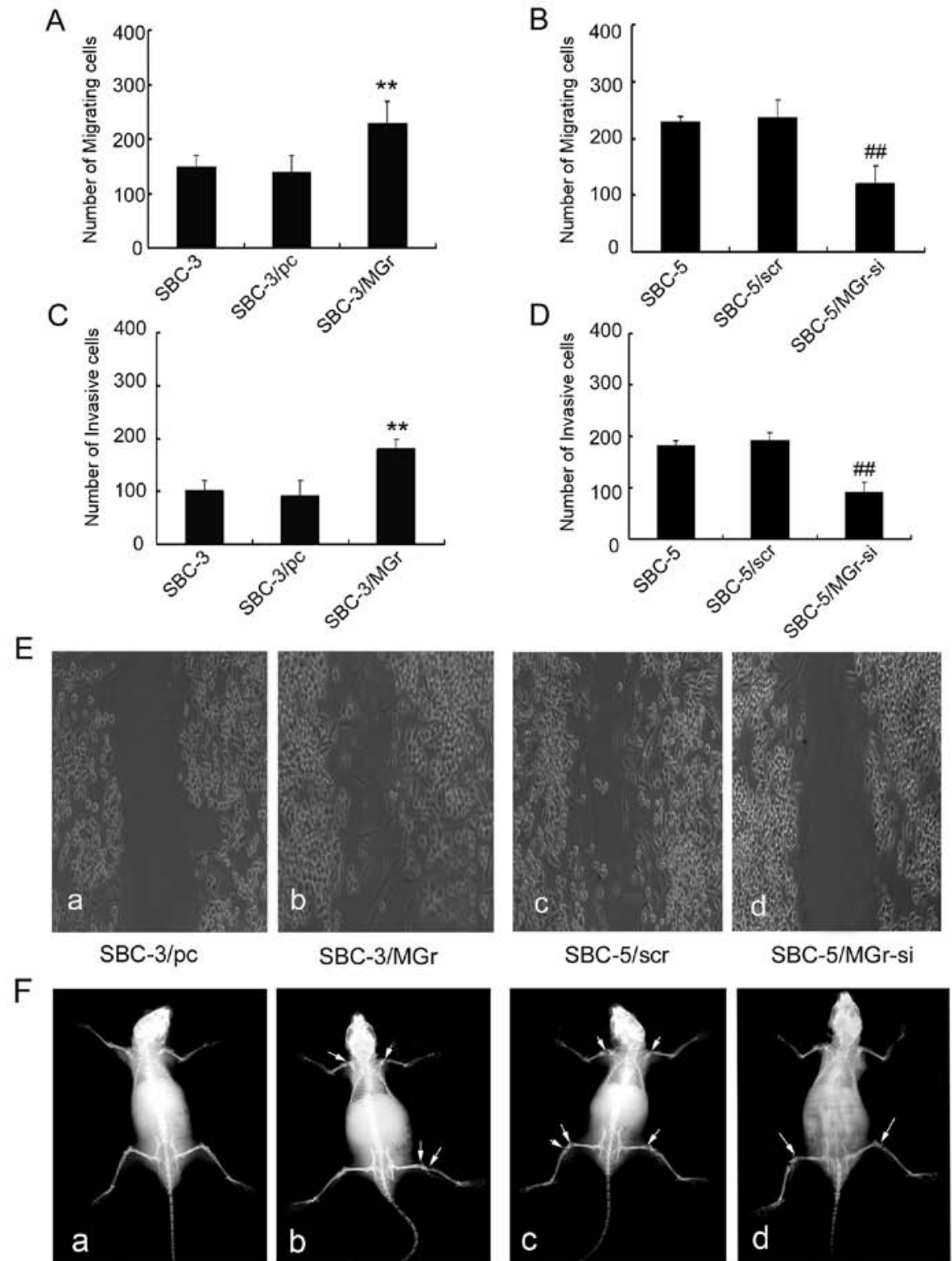

SBC-3/MGr

SBC-5/scr

SBC-5/MGr-si

SBC-3/pc
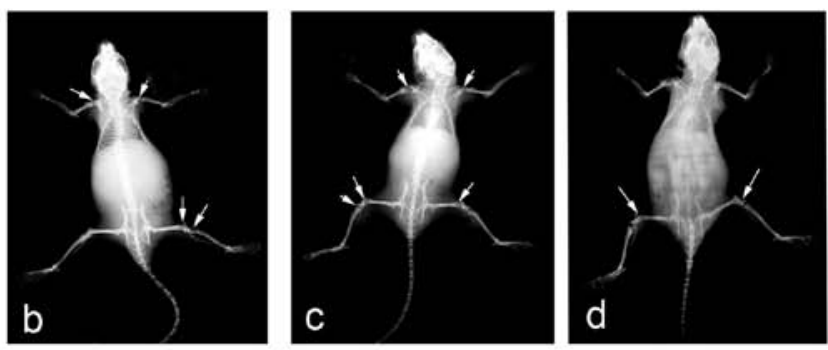

SBC-5/MGr-si

Figure 3. Effects of the upregulation or downregulation of MGr1-Ag in SCLC cells on cell invasion and migration in vitro and in vivo. (A and B) Migratory ability was evaluated by counting the number of cells that migrated through $8-\mu \mathrm{m}$ pore Transwell membranes ( ${ }^{* *} \mathrm{P}<0.01 \mathrm{vs}$. SBC-3/pc; ${ }^{\# \#} \mathrm{P}<0.01 \mathrm{vs}$. SBC-5/scr) $(\mathrm{C}$ and $\mathrm{D})$ Invasive ability was evaluated by counting the number of cells which invaded through Matrigel and $8-\mu \mathrm{m}$ pore Transwell membranes ( ${ }^{* *} \mathrm{P}<0.01 \mathrm{vs}$ SBC-3/pc; ${ }^{\# \#} \mathrm{P}<0.01$ vs. SBC-5/scr). (E) Wound healing assays were performed to detect the cell migratory capacity to repair the wound. (F) X-ray images of osteolytic bone metastatic lesions in the NOD/scid mice injected with $1 \times 10^{6}$ cells through the tail vein and sacrificed after 5 weeks. Arrows indicate the bone metastatic lesions.

complete loss of epithelial marker E-cadherin expression and high expression of mesenchymal marker fibronectin (Fig. 4B). In contrast, the same experiments were performed using SBC-5 cells. Immunofluorescence staining demonstrated that, compared to the SBC-5/scr cell line, the staining of E-cadherin was markedly increased in the SBC-5/MGr-si cell line whose MGr1-Ag expression was abolished by specific siRNA, while the staining of fibronectin was decreased (Fig. 5C). Data from the western blot analysis were consistent with data from the immunofluorescence staining in SBC-5 cells (Fig. 5B). Therefore, both the cell morphological and molecular changes observed in the SCLC cell lines indicated that EMT was associated with the occurrence of bone metastasis in SCLC, and Mgrl-Ag induced SCLC cells to undergo EMT.

\section{Discussion}

SCLC exhibits aggressive behavior including rapid growth and early spread to distant sites. Bone is one of the most frequent targets of SCLC metastasis. Bone metastasis leads to rapid deterioration in the quality of life of patients. However, effective curative therapy is limited in the clinical (2). Therefore, more basic studies concerning the mechanism of bone metastasis in SCLC are desperately needed. Previous research has indicated that bone metastases present as two types of lesions, osteoblastic or osteolytic, which mainly result from an imbalance between osteoblast-mediated bone formation and osteoclastmediated bone resorption (26). Numerous signaling pathways and various molecules are activated to drive this vicious 
A
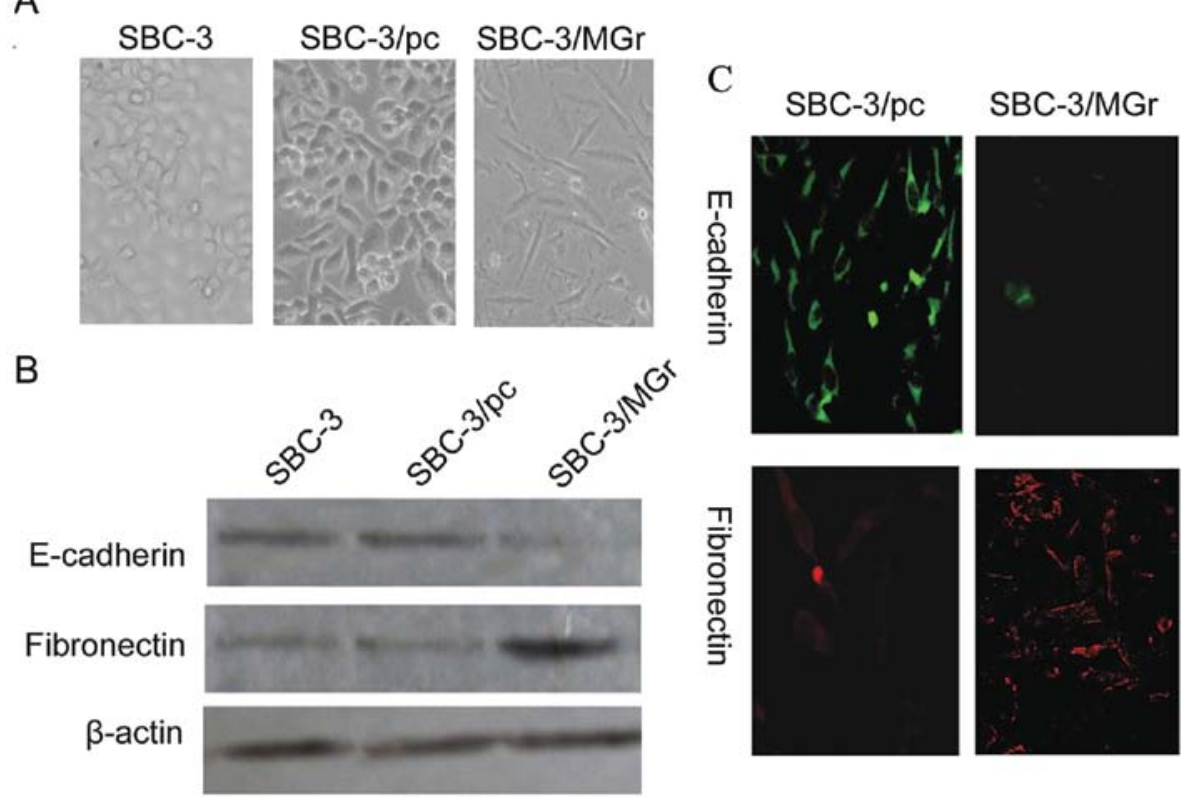

Figure 4. Upregulation of MGr1-Ag expression promotes SBC-3 cell EMT. (A) Morphology of SBC-3, SBC-3/pc and SBC-3/MGr cells was observed using a phase-contrast microscope and representative images are shown (x200 original magnification). (B and C) Epithelial marker (E-cadherin) and mesenchymal marker (fibronectin) in SBC-3, SBC-3/pc and SBC-3/MGr cells were detected by western blot analysis and immunofluorescence staining.

A
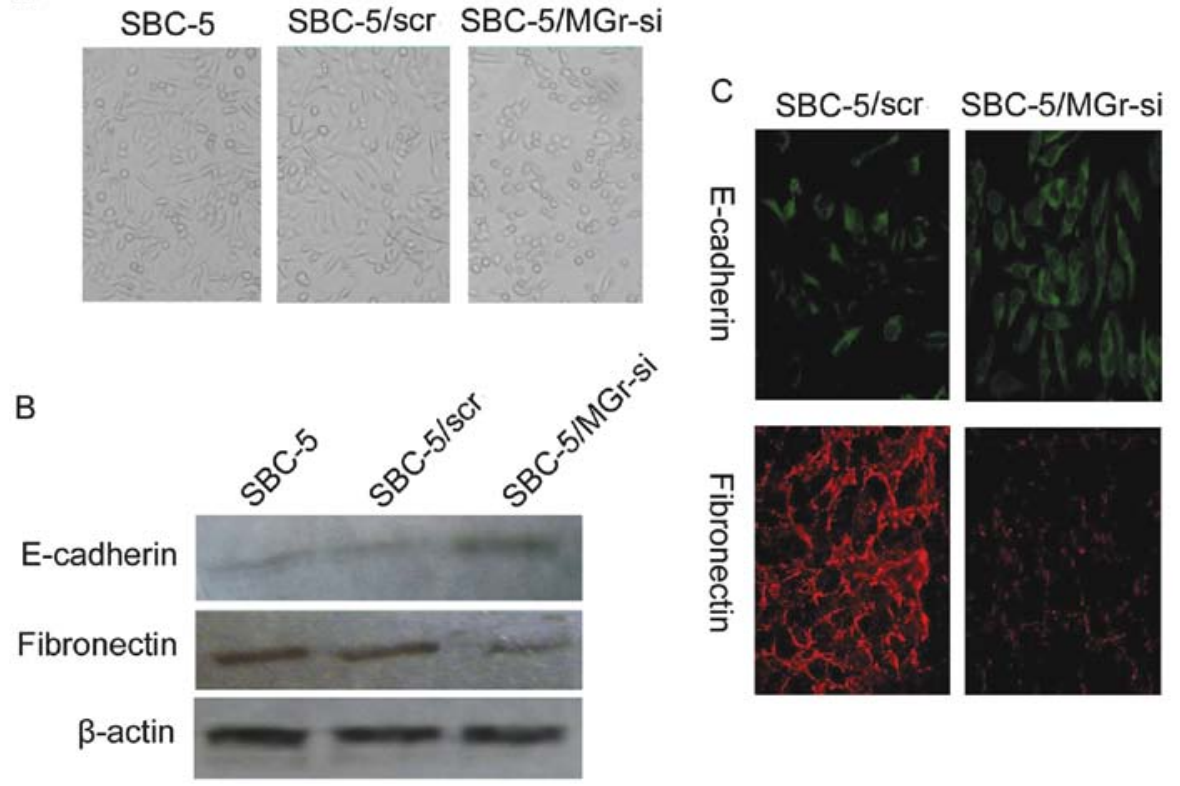

Figure 5. Inhibition of MGr1-Ag expression reverses EMT in SBC-5 cells. (A) Morphology of SBC-5, SBC-5/scr and SBC-5/MGr-si cells was observed using a phase-contrast microscope and representative images are shown (x200 original magnification). (B and C) Epithelial marker (E-cadherin) and mesenchymal marker (fibronectin) in SBC-5, SBC-5/scr and SBC-5/MGr-si cells were detected by western blot analysis and immunofluorescence staining.

imbalance in malignant disease $(27,28)$. In general, most of the basic research has focused on the relationship between tumor cells and the imbalance of osteoblasts and osteoclasts. Yet, the understanding of the mechanisms by which tumor cells attach and lodge in bone remains a void to be filled.

Malignant cell attachment and lodgment in bone are complex processes involving multiple interactions of tumor cells with host cellular and extracellular structures. One critical event is the formation of micrometastases. The attachment of cancer cells to basement membrane components is the first step in the metastatic process. This step may be mediated in part by specific cell-surface receptors which bind to laminin in the basement membrane. Notably, MGr1-Ag demonstrates high laminin-binding activity. Additionally, an increase in the expression of MGr1-Ag has been found in a variety of common cancers. In many cases, a positive correlation with 
the aggressiveness or metastatic potential has also been observed (16-19). Yet, whether MGr1-Ag plays a role in the bone metastasis of SCLC is unclear.

In the present study, we first detected the expression of MGr1-Ag in SBC-3 and SBC-5 cell lines, which have similar genetic background to human SCLC but different potential for bone metastasis (SBC-5 cells demonstrate a higher capability for promoting bone metastasis). Western blot analysis and real-time PCR showed that the expression of MGrl-Ag was markedly higher in SBC-5 cells than that in SBC-3 cells. Overexpression of MGr1-Ag was found to be associated with the high rate of bone metastasis in SCLC cells. Next, we upregulated the expression of MGr1-Ag in SBC-3 cells and observed the alterations in cellular behavior. We found that forced overexpression of MGrl-Ag in SBC-3 cells enhanced cell invasive and migratory abilities in vitro as well as increased bone metastatic lesions in vivo. Meanwhile, RNA interference targeting MGr1-Ag in SBC-5 cells partly abolished cell invasive and migratory abilities in vitro and decreased the number of bone metastatic lesions in vivo. Taken together, these findings suggest that MGr1-Ag functions to promote the invasion, migration and bone-metastatic ability of SCLC cells.

Recent research has shown a novel view relating metastasis with the heterogeneity of SCLC (23). This study found that tumors were often composed of phenotypically different cells with either a neuroendocrine marker or mesenchymal marker profile. Moreover, the crosstalk between mesenchymal and neuroendocrine cells strongly influenced their behavior. When engrafted as a mixed population, the mesenchymal cells endowed the neuroendocrine cells with metastatic capacity. These findings provide evidence that the poor prognosis of SCLC is due to the presence of cells with a mesenchymal character. Furthermore, in our research, variations in the cell morphology between SBC-3 and SBC-5 cells were observed. After transfection with the MGr1-Ag overexpression vector, the morphology of the SBC-3 cells changed from a round to a fusiform shape. Moreover, in the SBC-5 cells, the cell morphology was altered from fusiform to a round shape after the inhibition of MGr1-Ag expression with siRNA. These findings suggest that EMT was involved in the MGr1-Ag-mediated promotion of invasion, migration and bone-metastasis in SCLC cells. Thus, we detected the expression of epithelial marker (E-cadherin) and mesenchymal marker (fibronectin) in cells with different bone-metastatic potential and MGrl-Ag expression level. The results indicated that, in cells with high bone-metastatic potential and high MGrl-Ag expression level, the expression of the epithelial marker was decreased, and the expression of the mesenchymal marker was increased. In contrast, in cells with low bone-metastatic potential and low MGr1-Ag expression level, the levels of expression of the EMT markers were reversed. Thus, Mgrl-Ag likely promotes SCLC invasion and bone metastasis via the EMT pathway, but the precise role of EMT and its heterogeneity character in SCLC with bone metastasis still requires further study.

In conclusion, our present data strongly demonstrated that MGr1-Ag promoted invasion and bone metastasis in SCLC via the EMT pathway both in vitro and in vivo. MGr1-Ag is a promising therapeutic target for SCLC with bone metastasis. In regards to the inducers of EMT, microenvironmental factors such as hypoxia and growth factors such as epidermal growth factor and transforming growth factor- $\beta$ may induce the expression of certain EMT regulators, such as ZEB-1, directly or indirectly to prompt tumor invasion. The extent of the upregualation and downregulation of the epithelial and mesenchymal markers varies in different types of cancer cells and under different stimuli. The mechanisms underlying the role of MGrl-Ag in these processes require further study.

\section{Acknowledgements}

This study was supported by grants from the National Nature Science Foundation of China (nos. 81101765 and 81172011).

\section{References}

1. Jemal A, Bray F, Center MM, Ferlay J, Ward E and Forman D: Global cancer statistics. CA Cancer J Clin 61: 69-90, 2011.

2. Rodriguez E and Lilenbaum RC: Small cell lung cancer: past, present, and future. Curr Oncol Rep 12: 327-334, 2010.

3. Jackman DM and Johnson BE: Small-cell lung cancer. Lancet 366: 1385-1396, 2005.

4. van Meerbeeck JP, Fennell DA and De Ruysscher DK: Small-cell lung cancer. Lancet 378: 1741-1755, 2011.

5. Mundy GR: Metastasis to bone: causes, consequences and therapeutic opportunities. Nat Rev Cancer 2: 584-593, 2002.

6. Uy HL, Mundy GR, Boyce BF, et al: Tumor necrosis factor enhances parathyroid hormone-related protein-induced hypercalcemia and bone resorption without inhibiting bone formation in vivo. Cancer Res 57: 3194-3199, 1997.

7. Cai Z, Chen Q, Chen J, et al: Monocyte chemotactic protein 1 promotes lung cancer-induced bone resorptive lesions in vivo. Neoplasia 11: 228-236, 2009.

8. Han JH, Choi SJ, Kurihara N, Koide M, Oba Y and Roodman GD: Macrophage inflammatory protein-lalpha is an osteoclastogenic factor in myeloma that is independent of receptor activator of nuclear factor kappaB ligand. Blood 97: 3349-3353, 2001.

9. Miki T, Yano S, Hanibuchi M, Kanematsu T, Muguruma $\mathrm{H}$ and Sone S: Parathyroid hormone-related protein (PTHrP) is responsible for production of bone metastasis, but not visceral metastasis, by human small cell lung cancer SBC-5 cells in natural killer cell-depleted SCID mice. Int J Cancer 108: 511-515, 2004.

10. Li M, Zhou M, Gong M, et al: A novel animal model for bone metastasis in human lung cancer. Oncol Lett 3: 802-806, 2012.

11. Miki T, Yano S, Hanibuchi M and Sone S: Bone metastasis model with multiorgan dissemination of human small-cell lung cancer (SBC-5) cells in natural killer cell-depleted SCID mice. Oncol Res 12: 209-217, 2000.

12. Zhang H, Yano S, Miki T, et al: A novel bisphosphonate minodronate (YM529) specifically inhibits osteolytic bone metastasis produced by human small-cell lung cancer cells in NK-cell depleted SCID mice. Clin Exp Metastasis 20: 153-159, 2003.

13. Liu Y, Zhang N, Wang Y, et al: Zinc finger E-box binding homeobox 1 promotes invasion and bone metastasis of small cell lung cancer in vitro and in vivo. Cancer Sci 103: 1420-1428, 2012.

14. Liu Y, Zhang Y, Min J, et al: Calcineurin promotes proliferation, migration, and invasion of small cell lung cancer. Tumour Biol 31: 199-207, 2010.

15. Ma NQ, Liu LL, Min J, et al: The effect of down regulation of calcineurin $A \alpha$ by lentiviral vector-mediated RNAi on the biological behavior of small-cell lung cancer and its bone metastasis. Clin Exp Metastasis 28: 765-778, 2011.

16. Jaseja M, Mergen L, Gillette K, Forbes K, Sehgal I and Copié V: Structure-function studies of the functional and binding epitope of the human $37 \mathrm{kDa}$ laminin receptor precursor protein. J Pept Res 66: 9-18, 2005.

17. Shi Y, Zhai H, Wang X, et al: Multidrug-resistance-associated protein MGr1-Ag is identical to the human 37-kDa laminin receptor precursor. Cell Mol Life Sci 59: 1577-1583, 2002.

18. Annabi B, Currie JC, Bouzeghrane M, et al: Contribution of the $37-\mathrm{kDa}$ laminin receptor precursor in the anti-metastatic PSP94-derived peptide PCK3145 cell surface binding. Biochem Biophys Res Commun 346: 358-366, 2006. 
19. Liu L, Ning X, Sun L, et al: Involvement of MGr1-Ag/37LRP in the vincristine-induced HIF-1 expression in gastric cancer cells. Mol Cell Biochem 303: 151-160, 2007.

20. Liu L, Sun L, Zhang H, et al: Hypoxia-mediated up-regulation of MGr1-Ag/37LRP in gastric cancers occurs via hypoxiainducible-factor 1-dependent mechanism and contributes to drug resistance. Int J Cancer 124: 1707-1715, 2009.

21. Micalizzi DS, Farabaugh SM and Ford HL: Epithelialmesenchymal transition in cancer: parallels between normal development and tumor progression. J Mammary Gland Biol Neoplasia 15: 117-134, 2010.

22. Voulgari A and Pintzas A: Epithelial-mesenchymal transition in cancer metastasis: mechanisms, markers and strategies to overcome drug resistance in the clinic. Biochim Biophys Acta 1796: 75-90, 2009.

23. Calbo J, van Montfort E, Proost N, et al: A functional role for tumor cell heterogeneity in a mouse model of small cell lung cancer. Cancer Cell 19: 244-256, 2011.
24. Lois C, Hong EJ, Pease S, Brown EJ and Baltimore D: Germline transmission and tissue-specific expression of transgenes delivered by lentiviral vectors. Science 295: 868-872, 2002.

25. Zhang F, Wang Q, Ye L, Feng Y and Zhang X: Hepatitis B virus $\mathrm{X}$ protein upregulates expression of calpain small subunit 1 via nuclear factor-kappaB/p65 in hepatoma cells. J Med Virol 82: 920-928, 2010

26. Bogenrieder T and Herlyn M: Axis of evil: molecular mechanisms of cancer metastasis. Oncogene 22: 6524-6536, 2003.

27. Roodman GD: Biology of osteoclast activation in cancer. J Clin Oncol 19: 3562-3571, 2001.

28. Sone S and Yano S: Molecular pathogenesis and its therapeutic modalities of lung cancer metastasis to bone. Cancer Metastasis Rev 26: 685-689, 2007. 